\title{
¿QUÉ TÉCNICA ANESTÉSICA DEBEMOS UTILIZAR PARA LA PREVENCIÓN DE ALTERACIONES DEL NEURODESARROLLO EN LA POBLACIÓN PEDIÁTRICA?
}

What anesthetic technique should we use in order to prevent alterations in the neuro development over the pediatric population?

\section{Angélica María Laguna Proaños ${ }^{1-5-6}$, Pedro José Baquero Marín ${ }^{1-4-5-6}$, Diego Fernando Salazar Ocampo ${ }^{2-4}$, David Emilio Maldonado Benitez ${ }^{3-5}$.}

1. Médico Interno. Fundación Universitaria Autónoma de las Américas. Sede Pereira.

2. Médico Anestesiólogo. Departamento de Anestesiología y Reanimación. Fundación Universitaria Autónoma de las Américas. Sede Pereira.

3. Médico Pediatra. Departamento de Pediatría. Fundación Universitaria Autónoma de las Américas. Sede Pereira.

4. Semillero de Investigación Médica en Anestesiología y Dolor (SIMAD). Grupo GISCO. Fundación Universitaria Autónoma de las Américas.

5. Semillero de Investigación en Pediatría y Puericultura (SIPEP). Grupo GISCO. Fundación Universitaria Autónoma de las Américas.

6. Asociación de Sociedades Científicas de Estudiantes de Medicina de Colombia (ASCEMCOL).

7 Email de contacto: pedrobaquerom@gmail.com

\section{Resumen}

La decisión de administrar anestesia general en la edad infantil sigue siendo controversial. Este tema no solo ha generado un alto grado de preocupación en los profesionales de la salud por los neonatos y lactantes, sino también, por la edad escolar, siendo susceptibles a alteraciones durante el proceso de consolidación del aprendizaje y memoria, donde los anestésicos alteran el funcionamiento cerebral, provocando alteraciónes en la sinaptogénesis y neurodegeneración en diferentes áreas como la corteza visual primaria, cortezas temporales / somato sensoriales, la corteza frontal y el hipocampo . Esto asociado a algunos factores de riesgo como los fármacos $\mathrm{y} / \mathrm{o}$ dosis utilizadas para el procedimiento, tiempo de exposición, o condiciones propias del paciente.

Palabras Claves: anestesiología; trastornos del Neurodesarrollo; anestesia de conducción

\section{Abstract}

The decision to administer general anesthesia is childhood age remains controversial. East the issue has not only generated a high degree of concern in health professionals for Neonates and infants, but also, by the school age, being susceptible to alterations. during the consolidation process of earning and memory, where anesthetics They alter brain functioning, causing alterations in synaptogenesis and neurodegeneration in different areas such as. primary visual cortex, temporal cortices / sensory, the frontal cortex and the hippocampus. This is associated with some factors. of risk as the drugs and / or doses used for the procedure, exposure time, or own conditions of the patient.

Keywords: anesthesiology; neurodevelopmental disorders; anesthesia conduction 


\section{Señor Editor}

Aproximadamente 1.5 millones de recién nacidos son sometidos cada año a un procedimiento quirúrgico, a pesar de ser prácticas esenciales, la decisión de administrar anestesia general en la edad infantil sigue siendo controversial. Este tema no solo ha generado un alto grado de preocupación en los profesionales de la salud por los neonatos y lactantes, sino también, por la edad escolar, siendo susceptibles a alteraciones durante el proceso de consolidación del aprendizaje y memoria, donde los anestésicos alteran el funcionamiento cerebral, provocando alteraciónes en la sinaptogénesis y neurodegeneración en diferentes áreas como la corteza visual primaria, cortezas temporales / somato sensoriales, la corteza frontal y el hipocampo. Esto asociado a algunos factores de riesgo como los fármacos y/o dosis utilizadas para el procedimiento, tiempo de exposición, o condiciones propias del paciente ${ }^{1-3}$.

En respuesta a éstas inquietudes "the FDA's Anesthetic and Life Support Drugs Advisory Committee" analizó la situación y concluyó que debido a la escasa existencia de datos e investigaciones sobre este tema no es posible justificar un cambio en la práctica anestésica; es por esto que actualmente, se realizan exhaustivas investigaciones, como el estudio GAS, un estudio multicentrico, alétorizado y controlado que incluyó niños mayores de 60 semanas de edad, sometidos a herniorrafia inguinal bajo anestesia regional o anestesia general, en 28 hospitales de Australia, Italia, Estados Unidos, Reino Unido, Canadá, Holanda y Nueva Zelanda. Dicho estudio presentó resultados parciales a los dos años de seguimiento, en la cual el uso de por lo menos una hora de anestesia general con Sevoflurane no demostró un aumento del riesgo de resultados adversos en el neurodesarrollo en comparación con la anestesia regional despierto, sin embargo, se espera que los resultados completos sean conocidos después de los cinco años de seguimiento en el año 2018, y éstos ofrezcan una evidencia más clara, debido a que en los dos primeros años de seguimiento es difícil diagnosticar adecuadamente la presencia de ciertos trastornos neurológicos o neuroconductuales ${ }^{2-5}$.

Es importante que se tenga en cuenta, a pesar de que la mayoría de los estudios en animales concluyan los efectos neurodegenerativos relacionados con la exposición a la anestesia general a edades tempranas, se deberá evaluar riesgo - beneficio para el paciente, mientras se esclarece con más exactitud los riesgos relacionados en el neurodesarrollo asociados a la técnica anestésica general y regional ${ }^{4}$.

\section{Bibliografía}

1. Nasr VG, Davis JM. Anesthetic use in newborn infants: the urgent need for rigorous evaluation. Pediatr Res. julio de 2015;78(1):2-6.

2. Davidson AJ, Disma N, de Graaff JC, Withington DE, Dorris L, Bell G, et al. Neurodevelopmental outcome at 2 years of age after general anaesthesia and awake-regional anaesthesia in infancy (GAS): an international multicentre, randomised controlled trial. Lancet Lond Engl. 16 de enero de 2016;387(10015):239-50.

3. Lee JH, Zhang J, Wei L, Yu SP. Neurodevelopmental implications of the general anesthesia in neonate and infants. Exp Neurol. octubre de 2015;272:50-60.

4. Lönnqvist P-A, Ecoffey C, Bosenberg A, Suresh S, Ivani $G$. The European society of regional anesthesia and pain therapy and the American society of regional anesthesia and pain medicine joint committee practice advisory on controversial topics in pediatric regional anesthesia I and II: what do they tell us? Curr Opin Anaesthesiol. octubre de 2017;30(5):613-20.

5. McCann ME, Withington DE, Arnup SJ, Davidson AJ, Disma N, Frawley G, et al. Differences in Blood Pressure in Infants After General Anesthesia Compared to Awake Regional Anesthesia (GAS Study-A Prospective Randomized Trial). Anesth Analg. septiembre de 2017;125(3):837-45. 